\title{
Superior Mesenteric Vein
}

National Cancer Institute

\section{Source}

National Cancer Institute. Superior Mesenteric Vein. NCI Thesaurus. Code C33687.

A vein that follows the path of the superior mesenteric artery and joins with the splenic vein to form the hepatic portal vein. 